\title{
COMBINED OPTICAL AND X-RAY \\ OBSERVATIONS OF VARIABLE STARS
}

\author{
C. STUART BOWYER* \\ Mullard Space Science Laboratory, University College London, U.K.
}

\begin{abstract}
Two problems of X-ray astronomy are discussed: (1) Optical identifications of X-ray sources, and (2) Optical and X-ray observations of U Geminorum.

As X-ray astronomy matures, it has begun to interact more substantially with other more established fields of astronomy. The result of this interaction has usually been to stimulate both fields. In the past the X-ray astronomy group at Berkeley has endeavoured to utilize results from optical and radio astronomy to aid in the interpretation of our data and we have ourselves, upon occasion, obtained optical and radio data. We hope to increase our efforts in this regard in the future. In this vein I would like to discuss two topics in which we are currently involved which may be of particular interest to the IAU Symposium on Variable Stars.
\end{abstract}

\section{Optical Identifications of X-Ray Sources}

There are now a total of eight optically identified galactic X-ray sources (omitting SNR's), plus one which has an infrared counterpart but where interstellar extinction is too large to allow visible light observations (Cyg X-3). Of these eight, five are definitely established as binaries (Cyg X-1, Her X-1, Cen X-3, 3U 0900-40, $3 \mathrm{U} 1700-37$ ) while the nature of the remaining three (Sco X-1, Cyg X-2 and $3 \mathrm{U} 0614+09$ ) remains a mystery.

The importance of further optical identifications in extending our knowledge of the nature and distribution of compact X-ray sources cannot be over-estimated. Although published search efforts have been conducted to 17-18th magnitude, (e.g. Kunkel et al., 1970; Bahcall and Bahcall, 1975), none of the sources in the general direction of the galactic center (except Sco X-1) has yet been identified. Nevertheless, it has been generally assumed that these sources are in fact near the galactic center (except Sco X-1, with $d=250-500 \mathrm{pc}$ ). Based on this assumption, $X$-ray luminosity functions have been derived (Margon and Ostriker, 1973) and discussions of the stellar population with which these sources are associated have been frequent. Only through further optical identifications will these and other important questions be clarified. In addition to the unanswered questions about the collective properties of compact X-ray sources, there remains the problem of understanding the nature of individual objects of the Sco X-1 class. Despite intensive study of Sco X-1 itself, no real understanding of its nature has emerged.

* Permanent address: Berkeley Astronomy Department, University of California. 
A variety of criteria can be employed to secure the identification of an optical counterpart of an X-ray source once the search field has been made sufficiently small. The strongest possible confirmation can be made if the optical and X-ray source are found to be co-variable. One example of the type of confirmation is the optical identification of Her X-1 (2U $1705+34)$, a pulsating X-ray source in an eclipsing binary system, with the thirteenth-magnitude blue variable star HZ Hercules. Two of my students working with two other Berkeley students discovered the 1.7 day eclipse period and the $1.2 \mathrm{sec}$ pulsation period of this object making the identification conclusive (Davidsen et al., 1972). The 1.7 day eclipse period was discovered simultaneously by other workers (Bahcall and Bahcall, 1972) but confirmation of the $1.2 \mathrm{~s}$ pulsation period has not been made until recently (Groth, 1974).

Failing the detection of co-variability, identifications can be made on the basis of certain optical characteristics which seem to be common to the optical counterparts of X-ray objects such as UV excess, peculiar lines, and rapid variability. The strength of the resultant identification obviously depends upon how many peculiar objects lie within the error box.

The small error circle ( $2^{\prime}$ diameter) established for the source $3 U 0614+09$ by the University College London X-ray telescope on the Copernicus satellite provided an excellent opportunity to achieve another identification. Furthermore, the X-ray source was reported to be variable (Giacconi et al., 1974; Murdin et al., 1974) suggested a candidate optical star which they found to be variable. This lead a group of my students and co-workers at Berkeley to begin an optical study of the field of $3 \mathrm{U} 0614+09$ with simultaneous X-ray studies of the source being carried out by workers at the Mullard Space Science Laboratory of University College London.
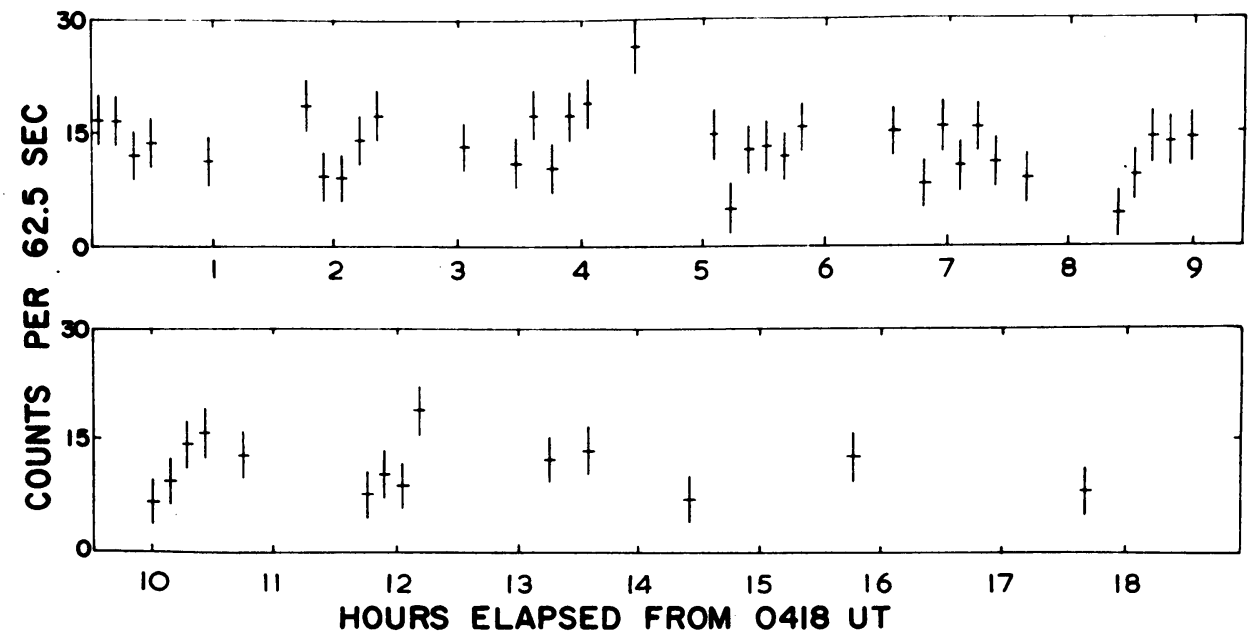

Fig. 1. Copernicus observations of 3 U $0614+09$ in February 8, 1974 from 0418 to 2258 UT. Each point is the background-subtracted sum of six 62.5-s integration periods, each separated by $24 \mathrm{~s}$ of deadtime. Gaps in the data are caused by target occultation or passage through the South Atlantic Anomaly. Error bars are $\pm 1-\sigma$ statistical errors, including source and background. 
The hope was, of course, that co-variability would be found, but a variety of optical data were obtained both as a means of studying the nature of the source and as an alternate means of providing an identification. The complete results of this work have been submitted for publication. (Davidsen et al., 1974) but let me summarise them here. A total of $36 \mathrm{~h}$ of X-ray observations were obtained with the Copernicus X-ray detector over the course of a week; a typical stretch of this data is shown in Figure 1. In brief, the source did not vary during the course of these observations and no evidence was found for the factor six flux variations noted by Giacconi et al. (1974). We must conclude that such large scale variability occurs only over time scales longer than several days, or else the source has quiescent periods as well as active ones. This obviously eliminated any hope of making an identification based on co-variability of the optical and $\mathrm{X}$-ray fluxes.

The optical results were more rewarding. The region containing $3 \mathrm{U} 0614+09$ was photographed with the $91-\mathrm{cm}$ Crossley reflector of Lick Observatory with band passes which approximated the $U$ and $B$ bands of the UBV photometric system. Values for $U-B$ ranged from +0.3 to +0.9 for all stars within the error circle except one which had $U-B=-0.5$. This star was, in fact, the candidate star suggested by

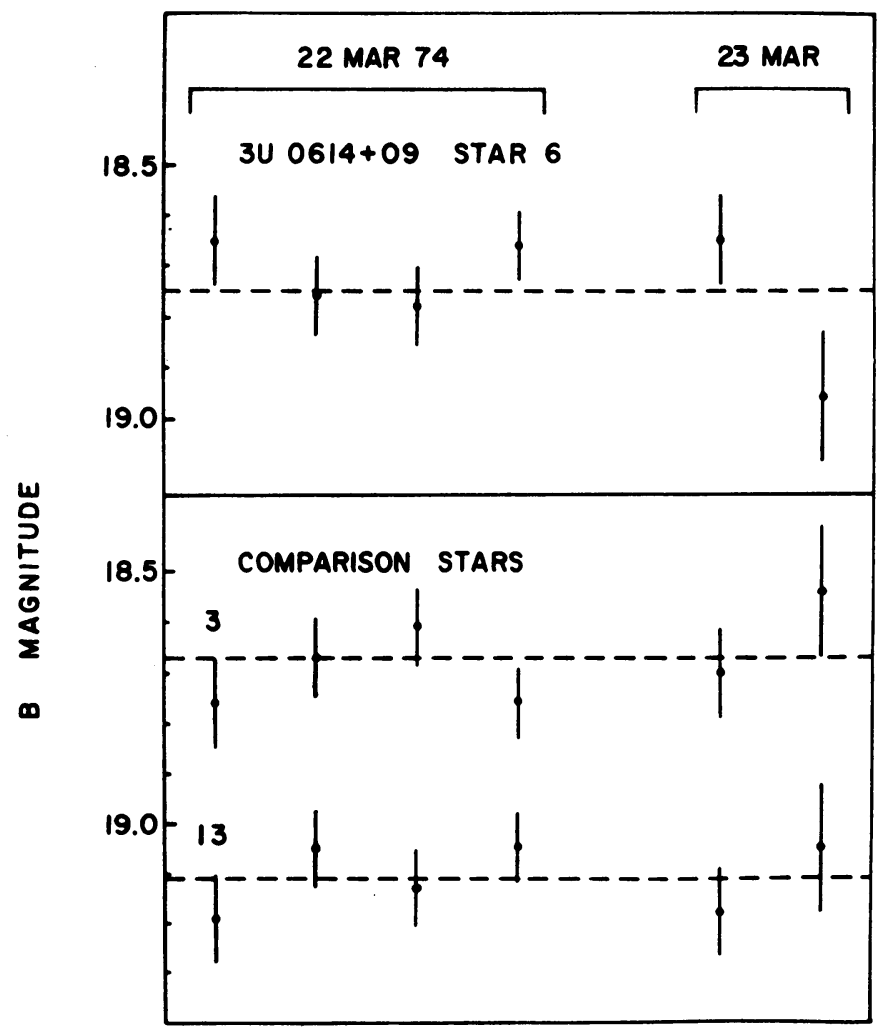

Fig. 2. Photographically derived $B$ magnitude for the optical counterpart of $3 U 0614+00$ and two comparison stars. The dashed lines are the average magnitude. Error bars are $\pm 1-\sigma$ random errors. There is no firm evidence of variability. 


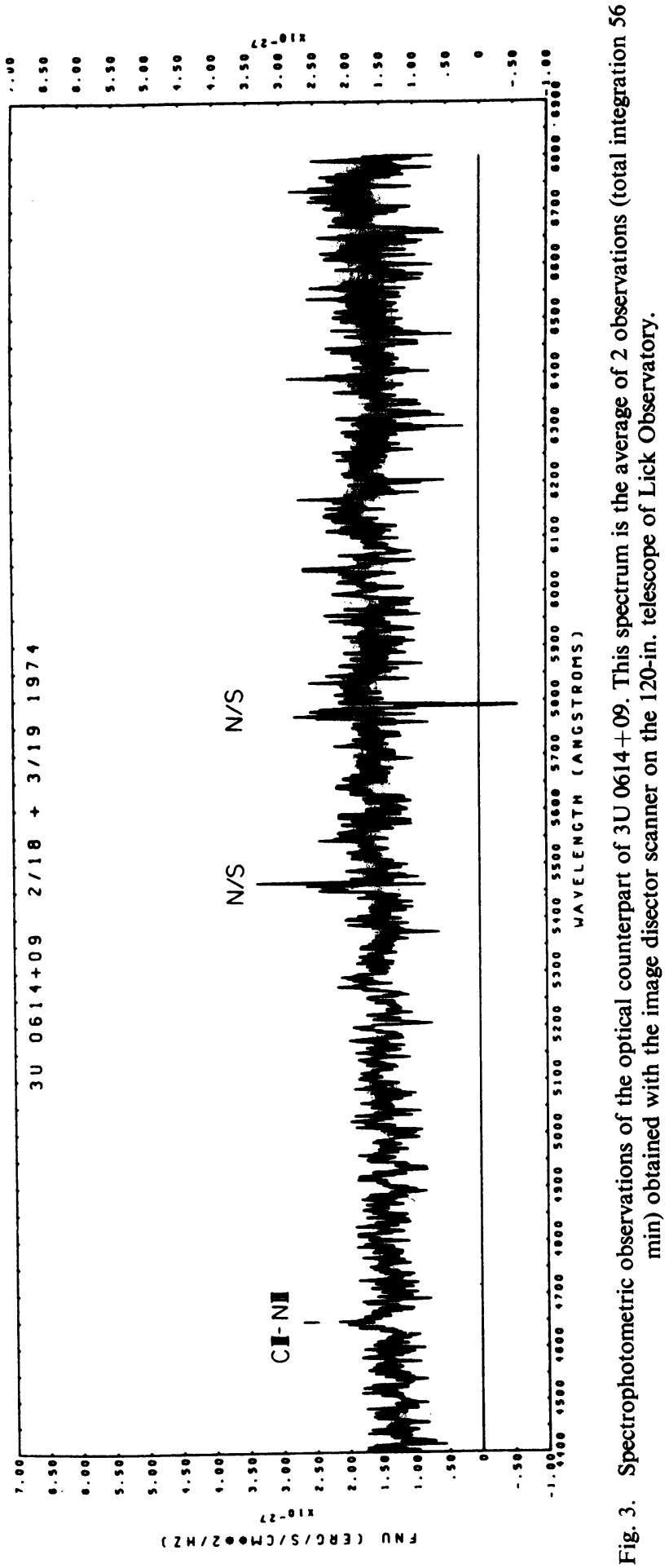


Murdin et al. (1974). Two additional types of data were taken of the candidate; the first concerned its variability, the second was its spectrum. A number of blue plates were taken over two nights with the Crossley reflector; the results are shown in Figure 2. The data do not provide positive evidence for variability, either internally or by comparison with the results of Murdin et al. The spectrum was obtained with the Robinson-Wampler image disector on the 3-m telescope of Lick Observatory. In Figure 3 is shown the resulting spectrum reduced to flux units (erg $\mathrm{cm}^{-2} \mathrm{~s}^{-1} \mathrm{~Hz}^{-1}$ ) above the Earth's atmosphere plotted against wavelength. The observed spectrum contains one strong feature, a broad emission line centered at $\lambda 4647$, which is identified as a blend of $N$ III 4640 and $C$ III 4650. The high excitation C III-N III blend is observed only in old novae, in hot stars with extended atmospheres, and in several optically identified X-ray sources, including Sco X-1.

The claim of identification, then, rests on the UV excess of the object and upon its unusual spectrum. While this identification is clearly not as strong as one based on optical and X-ray co-variability, it is nevertheless believed to be secure.

A case can be made that this object is similar in many respects to Sco X-1. Since considerable effort, both observational and theoretical, has been expended in the study of Sco X-1, it is encouraging to think that the results may have wider applicability. In addition, there are still many unanswered questions about Sco X-1, and some of these may only be answered by the study of other members of the same class.

\section{Optical and X-Ray Observations of $\mathbf{U}$ Geminorum}

The second topic I would like to discuss concerns work we have recently carried out on $U$ Geminorum. From a consideration of the energy liberated by the accretion process occurring in $U$ Geminorum variables, one expects that these systems should be soft X-ray-extreme ultraviolet emitters (Novikov and Thorne, 1973; Bath, 1973). My co-workers and I at Berkeley have recently carried out measurements of the soft $\mathrm{X}$-ray emission from this object (Henry et al., 1975) and although only upper limits to the flux were obtained, these were sufficiently stringent that important constraints can be placed on models of X-ray production in this system.

The data were obtained with two large area soft X-ray counters that were launched from White Sands Missile Range by a Black Brant VC rocket on 1973, February 10 at 0408 UT. Simultaneous optical observations (Mayall, 1974) indicated that U Gem was in a quiescent state at the time of this measurement. In our analysis the data employed were the number of counts detected in the 44 to $77 \AA$ band pass of our instrument rather than a flux limit since the conversion from counts to flux is somewhat dependent upon the shape of the assumed input spectrum. Unfortunately, the number of counts observed has little meaning without a detailed description of the instrumentation which would be inappropriate here. Hence to provide some feeling for the experiment $I$ have computed the upper limit to the flux observed on the basis of a black body spectrum of temperature $2 \times 10^{5} \mathrm{~K}$ attenuated by an interstellar hydrogen density of 0.25 atoms $\mathrm{cm}^{-3}$ averaged over the line of sight. With these assump- 
tions the 3- $\sigma$ upper limit to the flux in the 44 to $77 \AA$ band is $1.1 \times 10^{-10} \mathrm{erg} \mathrm{cm}^{2} \mathrm{~s}^{-1}$.

By utilising the work of Shakura (1973), Novikov and Thorne (1973), and Shakura and Sunyaev (1973) on the X-ray production in accreting binary systems, we can calculate the soft X-ray spectrum in emission from $U$ Gem as a function of the accretion rate of material onto the White Dwarf and a number of other parameters of the system. All of these other parameters can be reliably inferred from optical data, hence we can use our count rate limits to provide reasonably secure limits to the accretion rate onto the White Dwarf. The result is somewhat dependent upon the amount of intervening interstellar material which is assumed to be present, but the number is about $2 \times 10^{-8}$ solar masses $\mathrm{yr}^{-1}$ for reasonable column densities.

The period of $U$ Gem has been measured by a number of workers and is claimed by Warner and Nather (1971) to be increasing. Pringle (1975) has critically examined all the available data and finds the claim has been overstated. $\mathrm{He}$ and others have pointed out that among other things, Warner and Nather omitted a large amount of relevant data obtained by Krezminski (1965) and Paczynski (1965). The existing data show a period increase only at the $97.5 \%$ confidence level $(2.25 \sigma)$.

Let us explore for a moment the consequence of a period increase with the realisation that further work on this point is needed. It is not possible from the optical data alone to determine whether the period change is due to mass exchange or mass loss from the system. For either case the value deduced for the mass flow is about $3 \times 10^{-7}$ solar masses $\mathrm{yr}^{-1}$. From the upper limit to the accretion rate for $\mathrm{U}$ Gem obtained from our soft X-ray data, we see that most of the mass flow in U Gem is lost from the system. The accretion efficiency, defined as the fraction of matter that leaves the Roche lobe and accretes onto the compact object, is less than $10 \%$. This result may have relevance to binary $\mathrm{X}$-ray source models as well as to $\mathrm{U}$ Geminorum systems.

We at Berkeley expect to continue our work both on $U$ Gem systems and on the optical identification of X-ray sources. I hope that I can report more results on these topics in the not too distant future. For my part, I hope that additional optical data will be available on the question of the apparent period increase in U Gem.

\section{Acknowledgements}

The author would like to acknowledge financial support from an SRC Fellowship and support for travel from NASA grant NGR 05-003-450. He thanks Dr James Pringle for communicating his work before publication and Dr Bruce Margon and Mr Arthur Davidsen for useful discussions. Figures courtesy of American Astronomical Society.

\section{References}

Bahcall, J. and Bahcall, N. : 1972, Astrophys. J. 178, L1.

Bahcall, J. and Bahcall, N.: 1975, preprint.

Bath, G.: 1973, Nature Phys. Sci. 246, 84.

Davidsen, A., Henry, P., Middleditch, J., and Smith, H.: 1972, Astrophys. J. 177, L97. 
Davidsen, A., Malina, R., Smith, H., Spinrad, H., Margon, B., Mason, K., Hawkins, F., and Sanford, P.: 1974, Astrophys. J. 193, L25.

Giacconi, R., Murry, S., Gursky, H., Kellogg, E., Schreier, E., Matilsky, T., Kock, D., and Tananbaum, H.: 1974, Astrophys. J. Suppl. 27, 37.

Groth, E.: 1974, Astrophys. J. 192, 517.

Henry, P., Cruddace, R., Lampton, M., Paresce, F., and Bowyer, S.: 1975, Astrophys. J., 195, 107. Krezminski, W.: Astrophys. J. 142, 1051.

Kunkel, W., Osmer, P., Smith, M., Hoag, A., Schroeder, D., Hiltner, W., Bradt, H., Rappaport, S., and Schnopper, H.: Astrophys. J. 169, L169.

Margon, B. and Ostriker, J.: Astrophys. J. 186, 91.

Mayall, N.: 1974, private communication.

Murdin, P., Penston, M. J., Penston, M. V., Class, I., Sanford, P., Hawkins, F., Mason, K., and Willmore, P.: 1974, Monthly Notices Roy. Astron. Soc. 169, 23.

Novikov, I. and Thorne, K.: 1973, Les Houches Lectures on Black Holes.

Paczynski, B.: 1965, Acta. Astron. 15, 305.

Pringle, J.: 1975, Monthly Notices Roy. Astron. Soc. 170, in press.

Shakura, N.: 1973, Astron. Zh. 49, 921, 1972 = Soviet Astron. A.J. 16, 756, 1973.

Shakura, N. and Sunyaev, R.: 1973, Astron. Astrophys. 24, 337.

Warner, B. and Nather, R.: 1971, Monthly Notices Roy. Astron. Soc. 152, 219. 